\title{
A Full Virial Analysis of the Prestellar Cores in the Ophiuchus Molecular Cloud
}

\author{
Kate Pattle ${ }^{1}$ and Derek Ward-Thompson ${ }^{1}$ \\ on behalf of the JCMT Gould Belt Legacy Survey
}

\author{
${ }^{1}$ Jeremiah Horrocks Institute, University of Central Lancashire, Preston PR1 2HE, UK \\ email: kpattle@uclan.ac.uk, dward-thompson@uclan.ac.uk
}

\begin{abstract}
We use SCUBA-2, HARP $\mathrm{C}^{18} \mathrm{O} \mathrm{J}=3 \rightarrow 2$, Herschel and IRAM $\mathrm{N}_{2} \mathrm{H}^{+} \mathrm{J}=1 \rightarrow 0$ observations of the Ophiuchus molecular cloud to identify and characterise the properties of the starless cores in the region. The SCUBA-2, HARP and Herschel data were taken as part of the JCMT and Herschel Gould Belt Surveys. We determine masses and temperatures and perform a full virial analysis on our cores, and find that our cores are all either bound or virialised, with gravitational energy and external pressure energy on average of similar importance in confining the cores. There is wide variation from region to region, with cores in the region influenced by $\mathrm{B}$ stars (Oph A) being substantially gravitationally bound, and cores in the most quiescent region (Oph C) being pressure-confined. We observe dissipation of turbulence in all our cores, and find that this dissipation is more effective in regions which do not contain outflow-driving protostars. Full details of this analysis are presented by Pattle et al.(2015).
\end{abstract}

Keywords. stars: formation - ISM: clouds - ISM: kinematics and dynamics - ISM: individual objects: L1688 - submillimeter: ISM

\section{Observations and Data Processing}

We used SCUBA-2, HARP $\mathrm{C}^{18} \mathrm{O} \mathrm{J}=3 \rightarrow 2$ (White et al.2015), Herschel (Ladjelate et al., in prep) and IRAM N $\mathrm{H}^{+} \mathrm{J}=1 \rightarrow 0$ (André et al.2007) observations of the Ophiuchus molecular cloud to identify and characterise the properties of the starless cores in the region. The SCUBA2, HARP and Herschel data were taken as part of the JCMT (Ward-Thompson et al.2007) and Herschel (André et al.2010) Gould Belt Surveys. Full details of this analysis and the SCUBA-2 data are presented by Pattle et al.(2015).

We generated a set of convolution kernels from the SCUBA-2, SPIRE and PACS beam maps, following the method of Aniano et al.(2011). Using these kernels substantially decreases the discrepancies previously noted (Sadavoy et al.2013) between SCUBA-2 and Herschel fluxes. We also spatially filtered the Herschel maps to remove structure on scales greater than 600 arcsec, to which SCUBA-2 is not sensitive.

\section{Analysis and Discussion}

Seventy sources were identified in the SCUBA-2 $850 \mu \mathrm{m}$ map of L1688 using the curvaturebased CuTEx algorithm (Molinari et al.2011) in its detection mode. The sources were then fitted using a multiple-Gaussian fitting routine. Each SED was fitted with a modified black-body SED using the $160 \mu \mathrm{m}, 250 \mu \mathrm{m}, 450 \mu \mathrm{m}$ and $850 \mu \mathrm{m}$ bands, with a spectral index of 2.0. Mass estimates were derived from the fitted temperatures and $850 \mu \mathrm{m}$ flux densities. The sources were classified as starless cores or protostars based on morphology, temperature, PACS $70 \mu \mathrm{m}$ flux, and previous identifications. The mean HARP $\mathrm{C}^{18} \mathrm{O}$ and IRAM $\mathrm{N}_{2} \mathrm{H}^{+}$velocity dispersions were measured for each core for which data were available. Internal core energies were calculated from the gas velocity dispersion in $\mathrm{N}_{2} \mathrm{H}^{+}$-traced material $(\mathrm{T} \approx 7 \mathrm{~K}$; Stamatellos et al.2007), while the external pressure on the $\mathrm{N}_{2} \mathrm{H}^{+}$-traced material was determined from the gas velocity dispersion in $\mathrm{C}^{18} \mathrm{O}$ traced material. The virial stability of the cores, and the relative contributions of gravity and external pressure to core confinement, were estimated (Fig. 1).

All of our cores are either bound or virialised. Cores in the centre of Oph A (regions are as defined by Motte et al.1998) are typically strongly gravitationally bound. Cores in Oph C are 


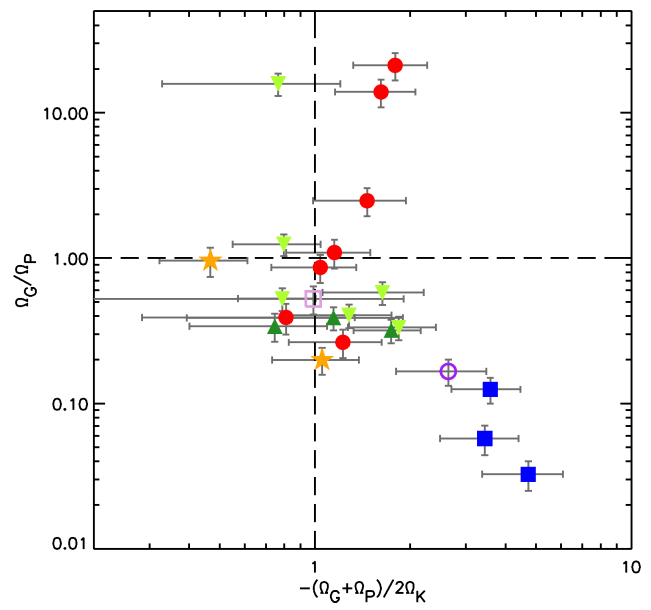

Figure 1. Core virial stability, against the ratio of gravitational potential energy to external pressure energy. Vertical dashed line: line of virial stability. The right-hand side of the plot is bound; the left-hand side, unbound. Horizontal dashed line: equipartition between external pressure energy and gravitational potential energy. Cores above the line are gravitationally bound; cores below the line are pressure-confined. Symbol coding as in Fig. 2.

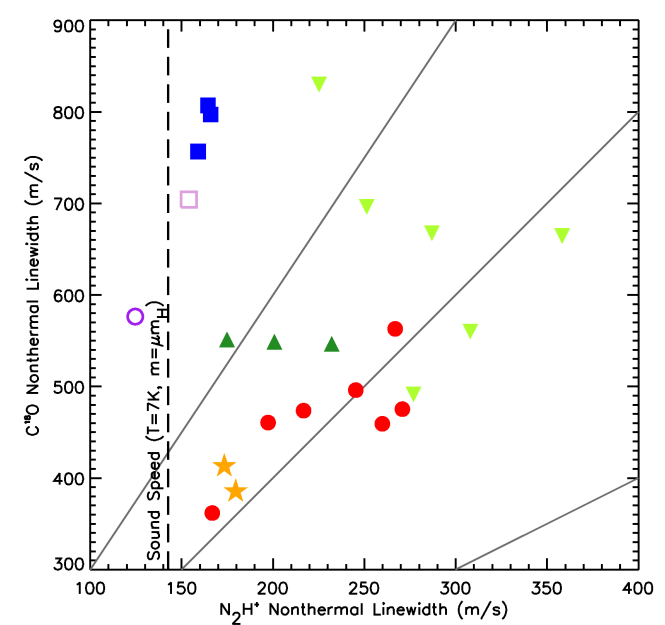

Figure 2. Comparison of non-thermal linewidths for $\mathrm{N}_{2} \mathrm{H}^{+}$and $\mathrm{C}^{18} \mathrm{O}$. Grey lines show the 1:1, 2:1 and 3:1 relations. Symbols denote region: Oph A - red closed circles; Oph A' - orange stars; Oph B1 - dark green upright triangles; Oph B2 - light green inverted triangles; Oph C - blue closed squares; Oph E - dark purple open circles; Oph F light purple open squares.

pressure-confined. The rest of the cores are in approximate equipartition between gravitational and pressure energy. We suggest that the influence of nearby B stars HD147889 and S1 might be sweeping up material in Oph A, causing higher densities. Oph $\mathrm{C}$ appears less evolved than the surrounding regions; we speculate that it it might be at a slightly different distance. Cores in Oph C, E and F dissipate turbulence more effectively than those in Oph A, A' and B (Fig. 2), which we hypothesise may be due to the lack of outflows in Oph C, E and F.

\section{References}

André, P., Belloche, A., Motte, F., Peretto, N. 2007, A\& A 472519

André, P., Men'shchikov, A., Bontemps, S., et al. 2010, A\&A 518102

Aniano, G., Draine, B. T., Gordon, K. D., Sandstrom, K. 2011, PASP 1231218

Evans, N. J. II, Dunham, M. M., Jørgensen, J. K. et al. 2009, ApJS 181321

Molinari, S., Schisano, E., Faustini, F. et al. 2011, A\&A 530133

Motte, F., André, P., Neri, R. 1998, A\&\&A 336150

Pattle, K., Ward-Thompson, D., Kirk, J. M., White, G. J. et al. 2015, MNRAS 4501094

Sadavoy, S. I., Di Francesco, J., Johnstone, D., et al. 2013, ApJ 767126

Stamatellos, D., Whitworth, A. P., Ward-Thompson, D. 2007, MNRAS 3791390

White, G. J., Drabek-Maunder, E., Rosolowsky, E. et al. 2015, MNRAS 4471996

Ward-Thompson, D., Di Francesco, J., Hatchell, J. et al. 2007, PASP 119855 